\title{
Set of measuring test fixtures for PCB materials research
}

\author{
Aleksei Rakov ${ }^{*}$, Vladimir Zhukov, Artem Kukanov, and Vladimir Ukhandeev \\ Joint-Stock Company “Research and Engineering Center ELINS”, R\&D Department, 124460, \\ Zelenograd, Moscow, Russia
}

\begin{abstract}
Designed and manufactured set of measuring test fixtures for printed circuit board (PCB) materials research and characterization is described in this article. Attention is paid to special designed test vehicle "General board" which includes six types of transmission lines that are used for PCB materials characterization by two measurement methods. Measurements of the frequency dependencies of the dielectric constant and dielectric loss for a number of the most widely used PCB substrates are presented. The comparison of the measurement results made by two measurement methods is also shown. The influence of the inhomogeneities of the PCB dielectric and some differences in the extracted material parameters data correlated with electro-magnetic fields structure in different transmission lines is demonstrated. In addition it is shown that measurements performed using "General board" can be used for characterization of the losses due to PCB surface plating.
\end{abstract}

\section{From prototyping to numerical simulation}

A process of electronic devices design has significantly changed and become more efficientwhen various numerical electromagnetic (EM) simulation tools have been involved in the research and development. This is illustrated by Fig.1.

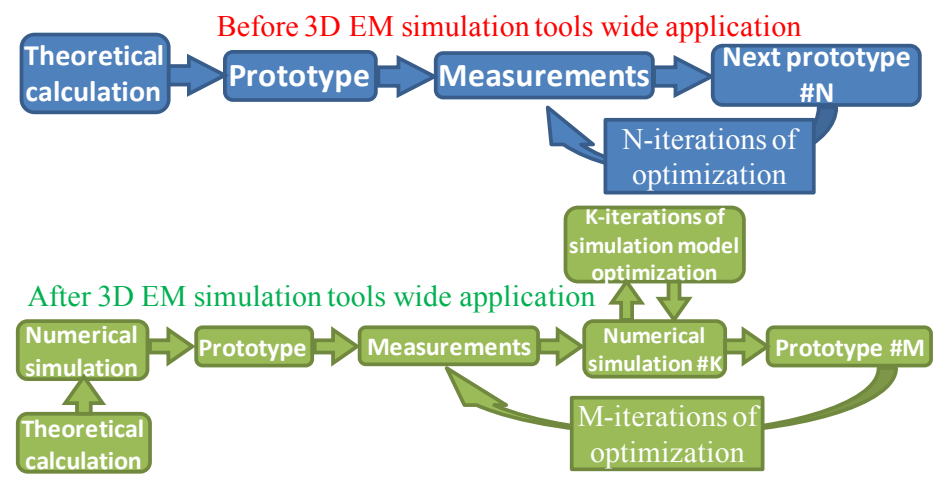

Fig. 1. Schematic representation of the electronics design process before and after wide application of the EM simulation.

* Corresponding author: a.rakov@elins.ru 
The major advantage of the new design process is that the number of iterations of the optimization (including prototype manufacturing) "M" is significantly less than " $\mathrm{N}$ ", which results in the prototype manufacturing time and costs decrease. However, the number of iterations of the numerical model optimization " $K$ " could be quite large that increases the costs of the required computational resources, as well as simulation time. New design process could not avoid the "prototype-measurements-prototype" conjugation, but it converts to "numerical simulation-prototype-measurements-numerical simulationprototype" conjugation. That is why the number " $\mathrm{K}$ " critically depends on numerical model adequacy to the manufactured prototype.

\subsection{Numerical model adequacy}

Numerical simulation model adequacy to the manufactured prototype depends on correctness of the 3D structure modelling and on the properly chosen material characteristics used in the simulation. A typical cross-section of a PCB and dielectric and conducting components in the stackup are schematically shown in Fig. 2.

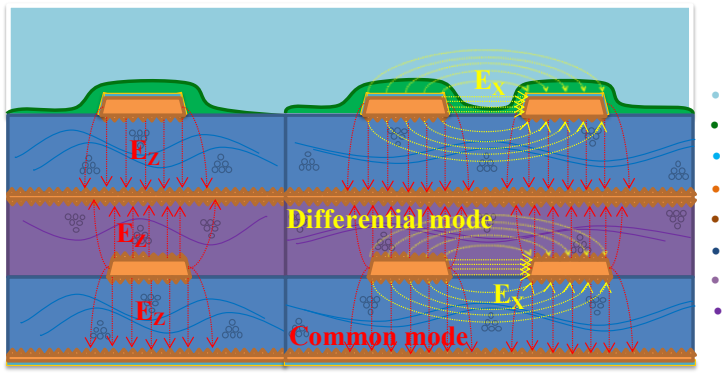

Potting compound

Solder mask

Conductor surface plating

Copper foil

Copper surface roughness

Core dielectric

Prepreg dielectric

Fiberglass weave

Fig. 2. Stackup PCB materials.

A PCB substrate is composed of core and prepreg dielectric materials that typically slightly differ, therefore, the dielectric substrate is inhomogeneous. For example typical PCB stackup uses RO4003C as a core and RO4450F as a prepreg. Core RO4003C and prepreg RO4450F have slightly different dielectric constant according to the datasheet 3.38@10GHz (3.55 - design DK) versus 3.52@10GHz (3.9 - design DK) and dielectric losses0.0027@10GHz versus0.004@10GHz. Thus, the effective dielectric characteristics for a PCB transmission line are typically somewhere in between RO4003C and RO4450F. If a designer has sufficient computational resources, it is worth to simulate the discrepancies in the core and prepreg characteristics. Designer also should extrapolate datasheet dielectric characteristics to the frequency range of work. If there is lack of computational resources one can use effective dielectric characteristics that could be measured experimentally.

\section{Set of measuring cells for PCB materials research}

A set of test fixtures for PCB materials research and characterization was designed and manufactured for wideband measurements on vector network analyser (VNA) to provide designers with accurate characteristics of PCB materials is shown in Fig. 3. A split cylinder resonator (SCR) for frequency range of $5-10 \mathrm{GHz}$ is shown at Fig. 3a. It is applicable for measuring dielectric parameters of bare laminates [1]. Gap coupled ring resonator is shown in Fig. 3b [2]. It can also measure dielectric parameters of the PCB laminates. Coaxial airfilled line that is shown in Fig. $3 \mathrm{c}$ is applicable for measurements of popping compounds using differential phase method [3] or solid dielectrics and magnetic materials using 
Nicolson-Ross-Weir (NRW) technique [4]. A specially designed test vehicle "General Board" that includes 6 types of the transmission lines for measurements by Stripline Sweep S-parameters (S3) method [5] and ring resonator method is shown in Fig. 3d. Measuring cell for ceramic substrate materials is shown in Fig. 3e. Note that currently there is no available multilayer PCB technology for ceramic substrates.

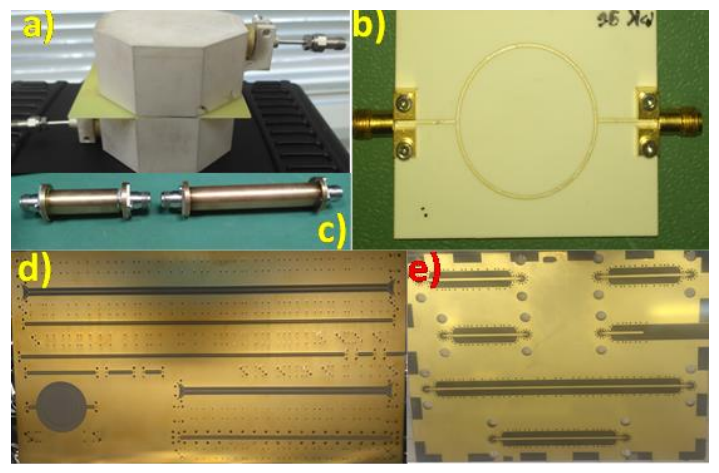

Fig. 3. Set of test fixtures for PCB material research and characterization.

\subsection{General board}

There is no solder mask in the General Board PCB stackup. Surface plating is comprised of immersion gold with nickel sublayer. The General Board topology includes single-ended and differential microstrips and striplines. Among them there are test-lines and auxiliary transmission lines for "through-reflect-line" (TRL) calibration pattern. Also, there are gap coupled microstrip and stripline ring resonators for the comparison of the extracted results with the ones by the S3-method. Note that there are mount holes for copper shield mounting around one of the single-ended microstrips. They are used for via-transition radiation loss research. The General Board allows to measure dielectric constant and tangent delta, estimate fiber weave effect, and investigate how field structure of the propagating modes in transmission lines affects the effective dielectric parameters.

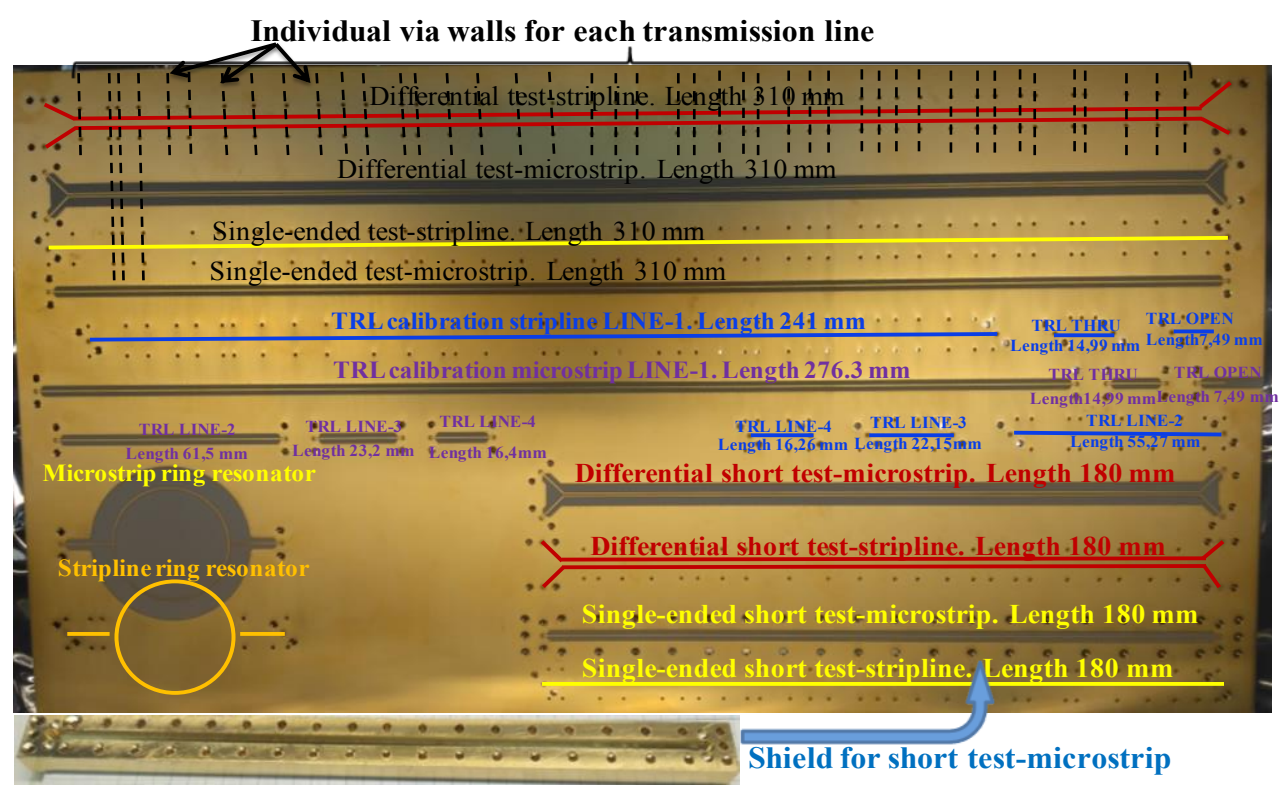

Fig. 4. General board topology. 


\section{Measurements}

Measurement results shown in Fig. 5 were obtained on the striplines of the General Board. The S3-method shows the uncertainties of the dielectric constant calculation around $2 \%$ and dielectric loss around 8\%. As it is indicated in Fig. 2, electric field lines of the differential mode are concentrated between signal traces. The traces of the differential stripline are immersed in the prepreg. Therefore, it is expected that the measured effective dielectric constant of the differential mode will be closer to the value of RO4450F. The measured dielectric parameters of the differential and common modes are almost the same. Dielectric parameters that are measured using ring resonator are very close to those measured on the single-ended stripline. Dielectric parameters that are measured on the one and another single-ended trace of the differential transmission line are slightly different that can be explained by the fiber weave effect. Measured dielectric constant and dielectric loss are slightly higher than values in the datasheet because of copper foil surface roughness influence. Surface roughness effect can be taken into account by the Differential Extrapolation Roughness Measurement (DERM) method [6].
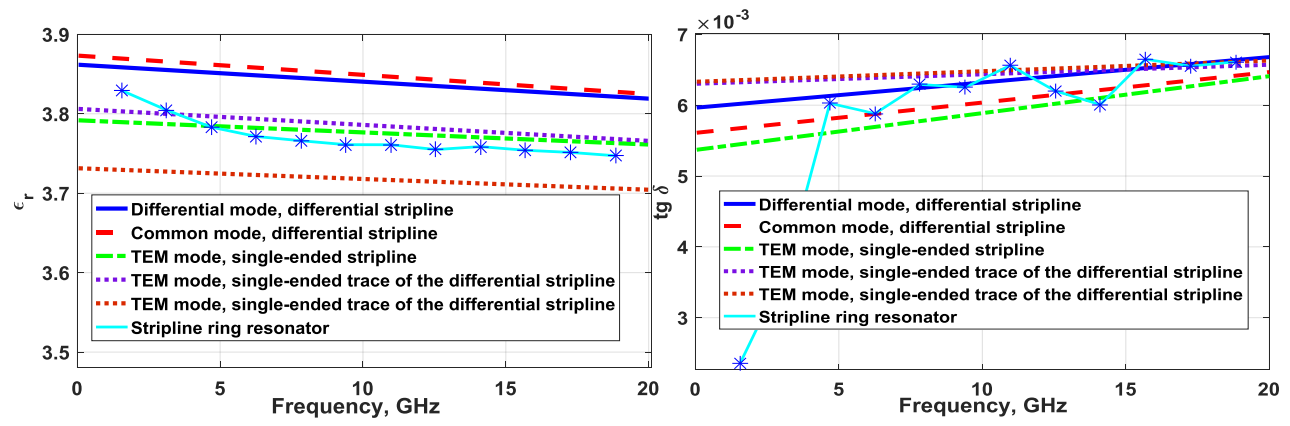

Fig. 5. Measured dielectric parameters on the different types of transmission lines and modes.

Measurements shown in Fig. 6 were performed on the microstrips of the General Board. The effective dielectric parameters are re-calculated to the substrate dielectric parameters using formulas in [7]. In Fig.2, one can see that if there is no solder mask on top of the microstrip structure, the electric field lines of the differential mode are concentrated in the air between signal traces. As is expected, the dielectric constant of the differential mode is much less than that of the common mode. (3.46 vs 3.94@1GHz). The dielectric constants that are measured using single-ended microstrip and gap coupled microstrip ring resonator are almost the same and close to the values from the datasheet.

The dielectric losses measured on the microstrips are three times higher than the measured on the striplines (Fig. 5). There is no radiation loss in the microstrip that was verified by S-parameters measurements with and without copper shield on the top of the transmission line. Thus, the possible reason is the sublayer of the nickel that is used for immersion gold surface plating. The nickel sublayer can be seen clearly in Fig.6 on the microstrip cross-section as a gray thin layer on the surface of the microstrip.

The substrates of the striplines and microstrips are almost the same on the same board, and copper foil surface roughness are also almost the same. Therefore, the loss due to nickel sublayer could be extracted using formulas (1)-(3). Losses in microstrip and stripline conductors can be calculated from the known signal trace geometry (Fig. 7) using formulas as in [7]. 

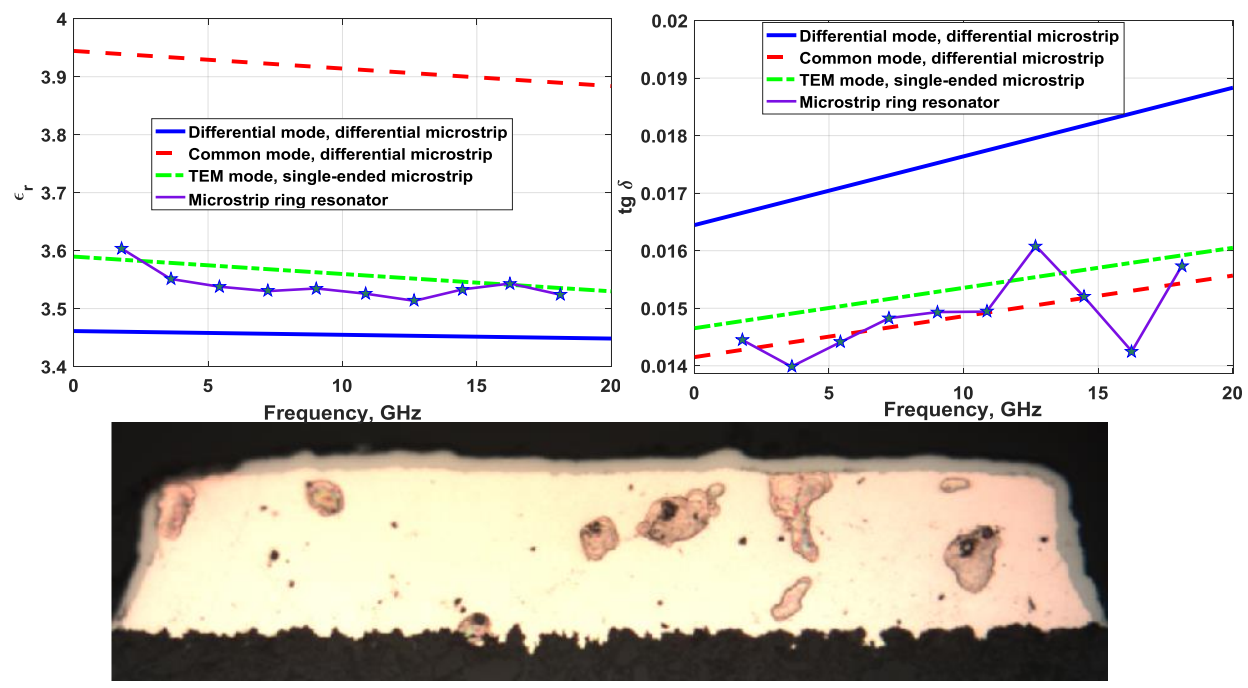

Fig. 6. Measured dielectric parameters on the different types of transmission lines and modes.

$$
\begin{gathered}
\alpha_{\text {total(stripline })}=\alpha_{\mathrm{c}(\text { smooth })}+\alpha_{\text {roughness }}+\alpha_{\text {substrate }} \\
\alpha_{\text {total(microstrip })}=\alpha_{\text {c(smooth })}+\alpha_{\text {plating }}+\alpha_{\text {roughness }}+\alpha_{\text {substrate }} \\
\alpha_{\text {plating }}=\alpha_{\text {total(microstrip })}-\left(\alpha_{\text {roughness }}+\alpha_{\text {substrate }}\right)-\alpha_{\text {c(smooth })}
\end{gathered}
$$

where $\alpha_{\text {total(stripline) }}$ is the total loss of the stripline, $\alpha_{\text {total(microstrip) }}$ is the total loss of the microstrip, $\alpha_{\mathrm{c}(\text { smooth) }}$ is the calculated loss in smooth conductor of the stripline, $\alpha_{c(\text { smooth) }}$ is the calculated loss in smooth conductor of the microstrip, $\alpha_{\text {roughness }}$ is loss due to surface roughness, and $\alpha_{\text {substrate }}$ is loss of the substrate.

The calculated frequency dependencies of the loss due to microstrip and stripline conductors and estimated loss due to nickel sublayer are shown in Fig. 7.

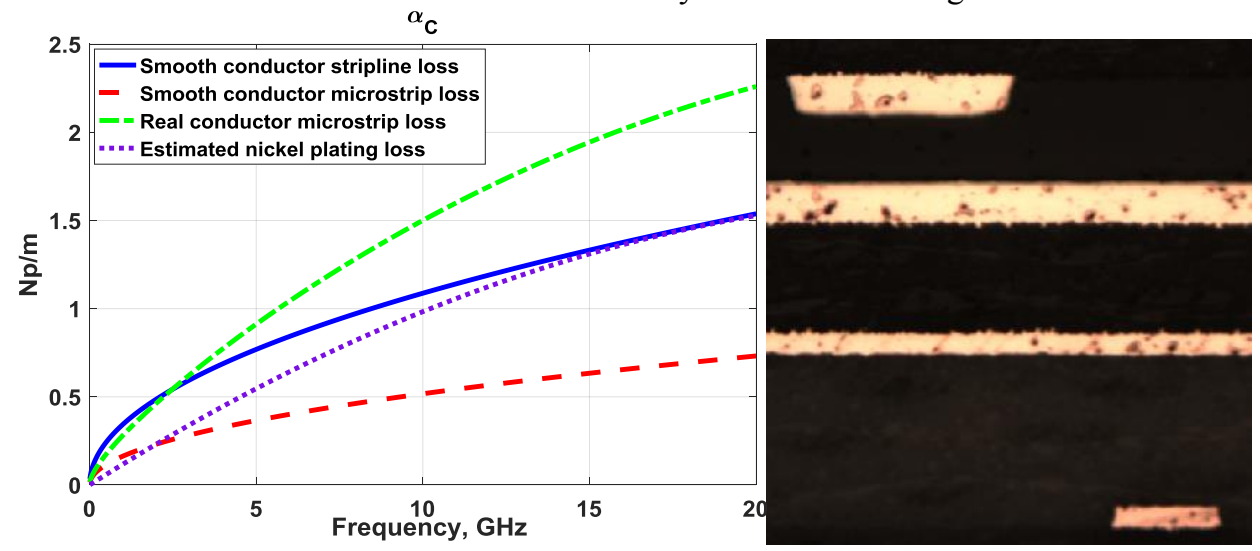

Fig. 7. Calculated microstrip and stripline conductor losses and extracted loss due to nickel sublayer.

\section{Conclusions}

Set of measuring test fixtures for PCB material research is designed and manufactured. General board test fixture allows obtaining accurate parameters of PCB substrate taking into account inhomogeneities of the substrate. The signal loss of the transmission line due to surface plating can be estimated from measurements performed using General Board. 


\section{References}

1. M. D. Janezic et. al., "Broadband Complex Permittivity Measurements of Dielectric Substrates using a Split-Cylinder Resonator", IEEE MTT-S International Microwave Symposium Digest (2004)

2. M. Kapoor, K.S. Daya, and G.S. Tyagi, "Coupled ring resonator for microwave characterization of dielectric materials." Special Issue on Surveillance Systems for Air Navigation Services, vol. 4, no. 2, pp. 241-246 (April 2012)

3. M. D. Janezic, J. A. Jargon, "Complex Permittivity Determination from Propagation Constant Measurements", IEEE Microwave and Guided Wave Letters, vol. 9 (1999)

4. J. Baker-Jarvis et. al., « Improved technique for determining complex permittivity with the transmission/reflection method», IEEE Transactions on Microwave Theory and Techniques, vol. 3 (1990)

5. M.Y. Koledintseva et. al., "Improved experiment-based technique to characterize dielectric properties of printed circuit boards," IEEE Trans. Electromag. Compat. (2013)

6. A. Koul, M.Y. Koledintseva, et. al., "Differential extrapolation method for separating dielectric and rough conductor losses in printed circuit boards", IEEE Trans. Electromag. Compat., vol. 54, no. 2, pp. 421-433 (Apr. 2012)

7. D.M. Pozar, Microwave Engineering, Wiley, $2^{\text {nd }}$ ed. (1998) 